\title{
Irrigation dynamic pressure-assisted hydrodissection during cataract surgery
}

\author{
This article was published in the following Dove Press journal: \\ Clinical Ophthalmology \\ 14 February 2017 \\ Number of times this article has been viewed
}

\author{
Yoichiro Masuda' \\ Hisaharu Iwaki \\ Noriko Kato' \\ Genichiro Takahashi' \\ Kotaro $\mathrm{Oki}^{3}$ \\ Hiroshi Tsuneoka ${ }^{4}$ \\ 'Department of Ophthalmology, The \\ Jikei University, Katsushika Medical \\ Center, ${ }^{2}$ Iwaki Eye Clinic, ${ }^{3}$ Oki Eye \\ Surgery Center, ${ }^{4}$ Department of \\ Ophthalmology, The Jikei University, \\ School of Medicine, Tokyo, Japan
}

\begin{abstract}
The irrigation dynamic pressure-assisted hydrodissection technique (irrigation-hydro $[\mathrm{iH}])$ does not require performing manual hydrodissection using a syringe and cannula to achieve cortical-capsular cleavage during cataract surgery. Since the iH technique uses the phaco tip to intentionally vacuum the intraocular fluid in order to induce the irrigation dynamic pressure for cortical-capsular cleavage, there is a reduction in the intraocular pressure (IOP) from the bottleheight-dependent hydrostatic pressure. Thus, since the peak irrigation pressure derived from the phaco tip sleeve will be limited by the height of the irrigation fluid bottle, this is advantageous in helping to avoid excessively high IOP during cortical-capsular hydrodissection. Using this technique, we were able to effectively perform phacoemulsification without complications in 607 of 609 cataract eyes. Our findings show that utilization of the iH technique would be of benefit to patients, as it prevents high-pressure hydrodissection-related complications, such as capsular block syndrome and tears in the anterior hyaloid membrane during cataract surgery.

Keywords: cataract surgery, hydrodissection, irrigation pressure, hydrodissection-related complication, capsular block syndrome, anterior hyaloid membrane tear, FLACS
\end{abstract}

\section{Introduction}

Modern phacoemulsification cataract surgery commonly uses manual cortical cleaving hydrodissection, which is performed via a syringe and cannula (manual-hydro). This technique requires the presence of an adequate injected fluid pressure in order to achieve the cortical-capsular cleavage. ${ }^{1-3}$ Intraocular pressure (IOP) during this procedure depends on several factors, including the volume of the injected fluid, the volume and type of the injected ophthalmic viscosurgical device (OVD), the incision size, and the surgeon's technique. Due to these factors, an excessively high pressure can occur within the endocapsular space, the capsule-zonule complex, and the posterior chamber-anterior hyaloid membrane barrier area, which can potentially induce severe complications. ${ }^{4-6}$ Complications associated with the high-pressure hydrodissection include capsular block syndrome, posterior capsular rupture, anterior capsule tear extension through the posterior capsule, tears in the anterior hyaloid membrane, and acute aqueous misdirection syndrome. ${ }^{5-14}$ Although there is a low rate of incidence, an occurrence can lead to more severe complications, such as endophthalmitis, retinal break, and retinal detachment. Therefore, surgeons need to carefully ensure that these complications do not occur.

The peak irrigation pressure derived from the phacoemulsification tip (phaco tip) sleeve is limited by the height of the irrigation fluid bottle (ie, hydrostatic pressure). Therefore, it is important to not exceed this hydrostatic pressure during the phacoemulsification cataract surgery. The bottle-height-dependent hydrostatic pressure can change in accordance with the 1) pressure loss in the irrigation line; 2) static (c)
hereby accept the Terms. Non-commercial uses of the work are permitted without any further permission from Dove Medical Press Limited, provided the work is properly attributed. For permission for commercial use of this work, please see paragraphs 4.2 and 5 of our Terms (https://www.dovepress.com/terms.php). 
pressure in the eye (IOP); and 3) dynamic pressure from the phaco tip sleeve during irrigation flows. Since the irrigation dynamic pressure is equal to the irrigation kinetic energy, this can potentially be used for cortical-capsular cleavage. Based on these known findings, we propose that the irrigation dynamic pressure could be utilized for cortical-capsular cleavage, thereby reducing the intraocular stress that is normally induced by high pressure. Thus, in this study, we describe a new technique for irrigation dynamic pressureassisted hydrodissection during cataract surgery, which we refer to as irrigation-hydro (iH).

\section{Patients and methods}

This study retrospectively reviewed the medical records of 609 consecutive eyes that underwent $\mathrm{iH}$ during phacoemulsification cataract surgery. The surgeries were performed by three different surgeons. The data recorded included age, gender, preexisting ocular pathology, ocular axial length, nuclear hardness, preoperative and postoperative best corrected visual acuities (BCVAs), intraocular lens (IOL) material and position, and information on any complications that occurred. The study was performed in accordance with the Declaration of Helsinki ethical principles for medical research involving human subjects. The study protocol was approved by the ethics committee of Jikei University School of Medicine. The patient's consent to review their medical records was not deemed necessary by the Institutional Review Board of Jikei University School of Medicine due to the retrospective nature of the study.

\section{Surgical techniques}

iH technique

The peak irrigation pressure derived from the phaco tip sleeve is limited by the irrigation fluid bottle height. Therefore, we hypothesized that the irrigation dynamic pressure from the tip of the sleeve could be used to cleave the cortical-capsular layer without causing an IOP that was excessively higher than the bottle-height-dependent hydrostatic pressure. In a preliminary study, we confirmed that the irrigation dynamic pressure derived from the phaco tip sleeve could cleave the porcine lens cortical-capsular layer when using the opensky method with a $50 \mathrm{~cm}$ bottle height, which is commonly utilized in phacoemulsification cataract surgery (Video S1). We created the $\mathrm{iH}$ technique as a way of replacing the manual-hydro technique. When using this technique during phacoemulsification cataract surgery, one or two stages are performed during the procedure (Figures 1 and 2).

\section{Phacoemulsification instrument parameters used for $\mathrm{iH}$ mode}

To ensure that the irrigation dynamic pressure is sufficient for achieving an effective cortical-capsular cleavage, the phaco instrument settings for $\mathrm{iH}$ are very important. The two different phaco machines used in this study were: 1) the Constellation Vision System (Alcon Laboratories, Inc., Fort Worth, TX, USA), which contains a $0.9 \mathrm{~mm}$ diameter phaco tip with a micro-sleeve and 2) the Centurion Vision System (Alcon Laboratories, Inc.), which contains a $0.9 \mathrm{~mm}$ diameter phaco tip with an ultra-sleeve. The phacoemulsification parameters used by the Constellation Vision System included the following: vacuum system, Venturi pump; vacuum pressure, $200 \mathrm{mmHg}$; irrigation pressure, $60 \mathrm{~cm} \mathrm{H}_{2} \mathrm{O}$; and ultrasound power, 0 . The phacoemulsification parameters used by the Centurion Vision System included the following: vacuum system, peristaltic pump; vacuum pressure, $350 \mathrm{mmHg}$; irrigation pressure, $36 \mathrm{mmHg}$; aspiration flow rate, $45 \mathrm{cc} / \mathrm{min}$, and ultrasound power, 0 .
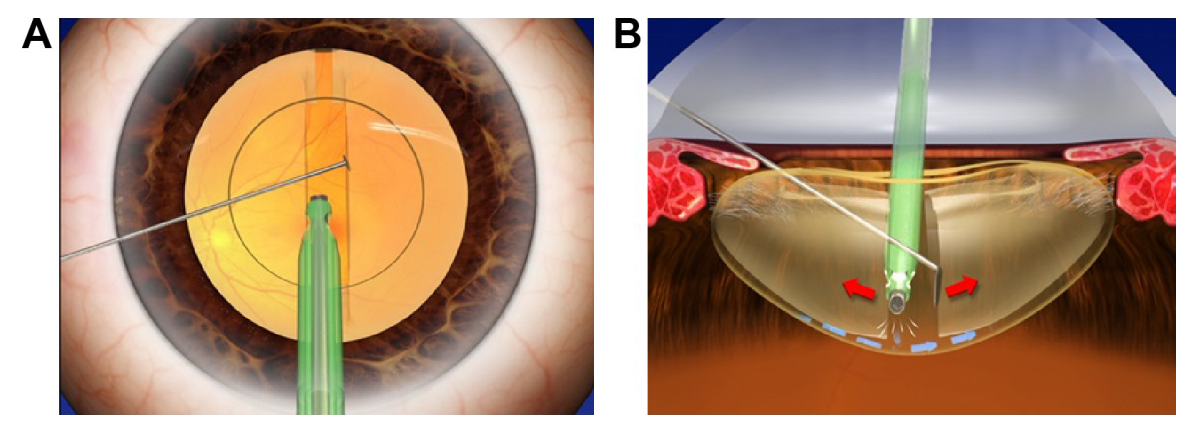

Figure I Posterior iH technique.

Notes: After the phaco tip is passed under the anterior capsule within the possible range, a vertical groove is sculpted in accordance with the divide and conquer method (A). The phacoemulsification tip exerts centrifugal force from the vertical groove sculpture on the base of the left-hand wall of the trench and from the hook on the base of the right-hand wall of the trench (cross action) with vacuuming of intraocular fluid by the phaco tip. This induces the irrigation flow from the phaco tip sleeve into the posterior cortical-capsular layers (B). As a result, the vertical groove sculpture is cracked into two hemispheres, with the irrigation dynamic pressure from the tip sleeve used to cleave the posterior cortical-capsular layer. The procedure is then repeated at several points on the vertical groove sculpture, with inversion of the phaco tip and the hook position (non-cross action) making it possible to complete the posterior cortical-capsular cleavage. The schematic illustration shows the direction of the movement of the lens segments caused by the instruments (red arrows), the vacuuming of the aqueous (white lines), and the irrigation flow (blue arrows).

Abbreviation: $\mathrm{iH}$, irrigation-hydro. 

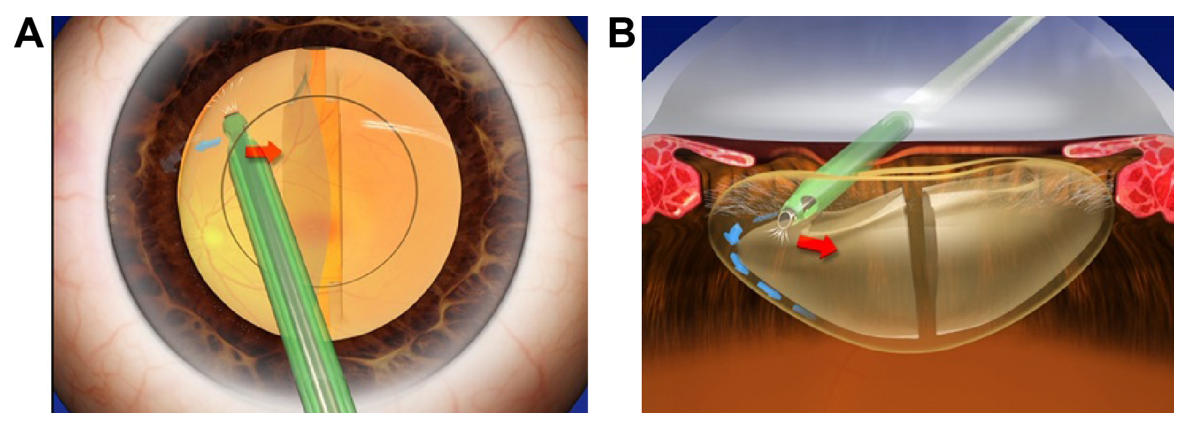

Figure 2 Anterior iH technique.

Notes: Following the placement of the phacoemulsification tip under the capsulorhexis edge, the aqueous humor is vacuumed and/or the side-port is depressed to induce irrigation flow into the anterior cortical-capsular layer (A). Subsequently the irrigation dynamic pressure is used to cleave the anterior, equatorial cortical-capsular layers (B). The schematic illustration shows the direction of the movement of the lens segments caused by the instruments (red arrows), the vacuuming of the aqueous (white lines), and the irrigation flow (blue arrows).

Abbreviation: $\mathrm{iH}$, irrigation-hydro.

\section{Posterior iH technique}

After the phaco tip is passed under the anterior capsule within the possible range, the phaco tip is then used to sculpt a vertical groove (Figure 1A). Once the sculpturing of the vertical groove is completed, centrifugal force is exerted by the phacoemulsification tip on the base of the left-hand wall of the trench and by the hook on the base of the right-hand wall of the trench (referred to as cross action), with vacuuming of the intraocular fluid by the phaco tip. In addition, there is induction of the irrigation flow from the phaco tip sleeve into the posterior cortical-capsular layers (Figure 1B). As a result of the vertical groove sculpture, the two hemispheres are cracked into two and the irrigation dynamic pressure from the tip sleeve causes cleavage of the posterior corticalcapsular layer. Through repetition of this method at several different points along the vertical groove sculpture, along with further repetitions after inverting the tip and hook position (referred to as non-cross action), these actions make it possible to achieve complete posterior $\mathrm{iH}$. In soft cataract surgery cases, however, this stage is not required.

\section{Anterior iH technique}

Following placement of the phaco tip under the capsulorhexis edge, the intraocular fluid is vacuumed, which induces the irrigation flow into the anterior cortical-capsular layer. The induced irrigation dynamic pressure from the phaco tip sleeve then cleaves the anterior, equatorial cortical-capsular layers (Figure 2A and B). One of the beneficial options of this technique is that the irrigation flow from the tip sleeve can be induced by depressing the side-port by a hook, with the irrigation flow able to compensate for the loss of intraocular fluid.

Cortical cleavage can be effectively performed using this one- or two-stage $\mathrm{iH}$ technique. The following section describes the actual surgical procedure.

\section{iH during phacoemulsification cataract surgery Posterior iH following vertical groove sculpture}

After completion of the initial steps of the cataract surgery (incision, OVD injection, and continuous curvilinear capsulorhexis $[\mathrm{CCC}]$ ), the phaco tip is inserted into the anterior chamber without performing the manual-hydro procedure. After the phaco tip is passed under the anterior capsule and the anterior capsular-connection is separated within the possible range (Figure 3A), a conventional vertical groove is sculpted in accordance with the divide and conquer method (Figure 3B). Subsequently, posterior iH is performed sequentially at both ends and at the center of the vertical groove sculpture (Figure 3C). When performing the posterior $\mathrm{iH}$, we found that the bottom of the vertical groove sculpture was the best place to use when completing the procedure. The vertical groove sculpture is cracked into two hemispheres, and the irrigation dynamic pressure from the tip sleeve is used to cleave the posterior corticalcapsular layer (Figure 3C). For effective posterior iH, the side hole of the sleeve is directed forward onto the posterior capsule, thereby making it possible for the irrigation flow to effectively cleave the posterior capsular connection. As a result, the posterior cortical-capsular connection can then be separated. If the anterior cortical-capsular connection is also separated using just this procedure, the anterior $\mathrm{iH}$ can be skipped, with the lens then freed from the cortical-capsular connection and rotated circumferentially by the phaco tip and a hook for removal. Videos $\mathrm{S} 2$ and $\underline{\mathrm{S} 3}$ present this technique in detail.

\section{Anterior $\mathrm{iH}$ following posterior $\mathrm{iH}$ (if needed)}

Anterior iH is performed after the posterior $\mathrm{iH}$, if needed (Figure 4). After placing the phaco tip under the left capsulorhexis edge, the anterior cortical-capsular iH is 

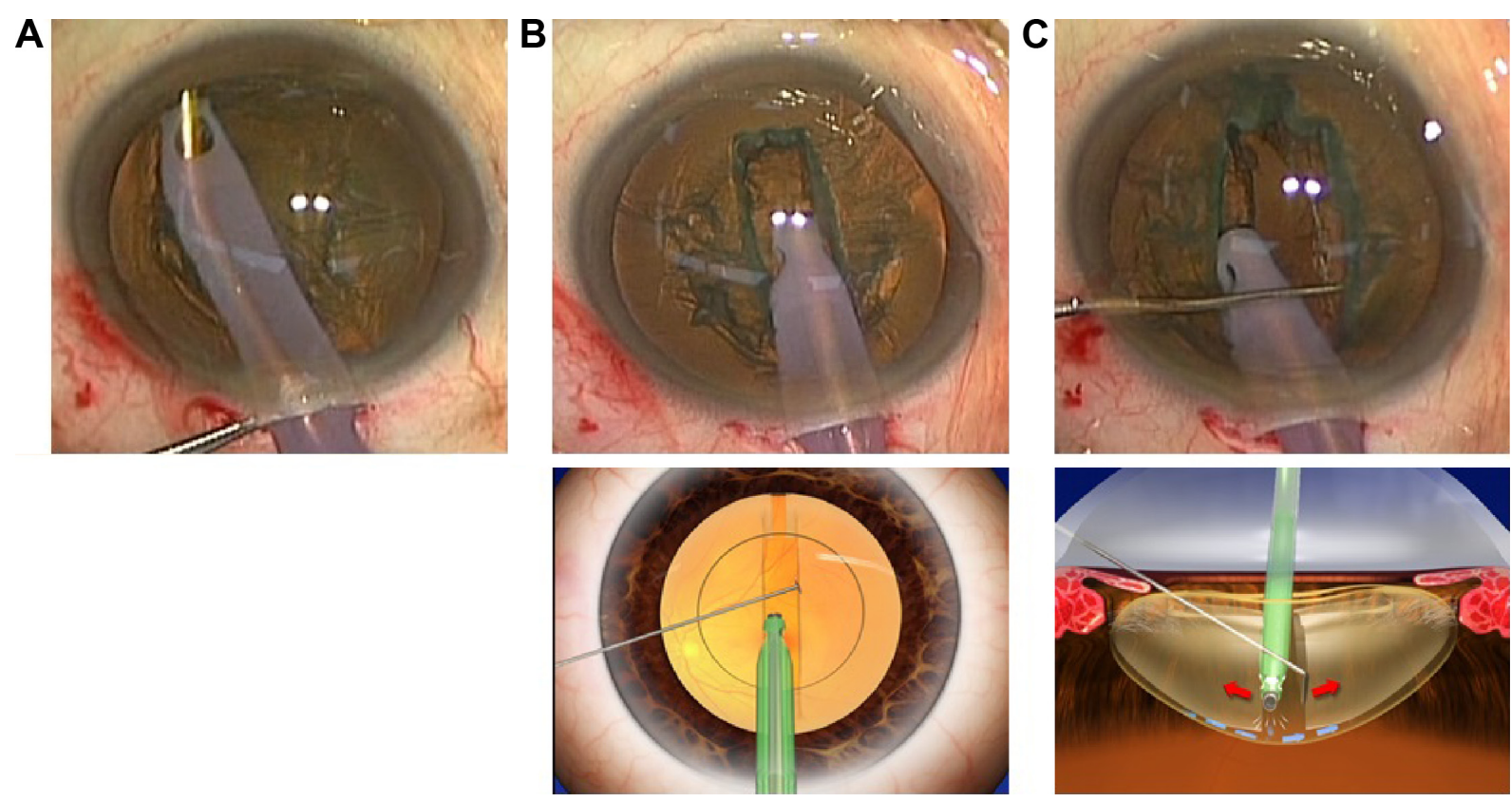

Figure 3 Posterior iH after sculpting of the vertical groove.

Notes: (A) The phaco tip is passed under the anterior capsule within the possible range. (B upper and lower) Conventional vertical groove sculpture. (C upper and lower) Posterior iH is performed sequentially at both ends and at the center of the vertical groove sculpture. As a result, this leads to the separation of the posterior cortical-capsular connection. If the anterior cortical-capsular connection can also be separated by using this procedure, the anterior iH technique does not need to be performed, and the lens can be freed from the cortical-capsular connection by using the phacoemulsification tip to circumferentially rotate it for removal by a hook. The schematic illustration shows the direction of the movement of the lens segments caused by the instruments (red arrows), the vacuuming of the aqueous (white lines), and the irrigation flow (blue arrows).

Abbreviation: $\mathrm{iH}$, irrigation-hydro.

performed on the left hemi-nucleus (Figure 4A) to free it from the cortical-capsular connection. Subsequently, anterior cortical-capsular iH is performed on the right hemi-nucleus (Figure 4B), which is then also freed. Cortical-capsular separation is now complete and the lens can be rotated circumferentially (Figure 4C). Videos S2 and $\underline{\mathrm{S} 3}$ present the technique in detail.

The remainder of the procedure, including the cataract removal and IOL implantation, is then performed in a normal manner.

\section{Results}

The patients included 352 females and 257 males, with an average age of 72.9 years old (range 32-93 years). Anterior ocular segment conditions present at surgery included pseudoexfoliation syndrome in 17 eyes, phacodonesis in 15 eyes, inadequate mydriasis in 45 eyes (moderate: 39 eyes, poor: 6 eyes), shallow anterior chamber in 13 eyes, corneal opacity in 3 eyes, previous traumatic eye in 1 eye, anterior capsule calcification in 4 eyes, posterior polar cataract in 9 eyes, post-laser iridotomy in 6 eyes, and intraoperative floppy iris syndrome in 1 eye. Posterior ocular segment conditions present at surgery included diabetic retinopathy in 10 eyes, age-related macular degeneration (AMD) in 11 eyes, glaucoma in 20 eyes, epiretinal membrane in 10 eyes, chorioretinal atrophy in 1 eye, and a previously vitrectomized eye in 2 eyes. The ocular axial length was $<21.5 \mathrm{~mm}$ in 10 eyes, $21.5-26 \mathrm{~mm}$ in 556 eyes, $26-30 \mathrm{~mm}$ in 42 eyes, and $>30 \mathrm{~mm}$ in 1 eye. The nucleus hardness was grade 1 or softer in 22 eyes, grade 2 in 285 eyes, grade 3 in 274 eyes, and grade 4 or harder in 28 eyes.

A polyacrylic IOL was implanted in 609 eyes, including 606 IOLs implanted in the bag and 3 IOLs implanted out of the bag. In 6 eyes with poor mydriasis, the pupil was dilated using a Malyugin pupil expansion device or sphincterotomy.

Posterior capsule rupture occurred in 2 eyes $(0.3 \%)$. In both cases, the IOL was implanted out of the bag and the final BCVA was better than the preoperative BCVA. Although a CCC tear occurred in 4 eyes, iH and phacoemulsification were successfully performed in all of the eyes without expansion of the tear to the posterior capsule.

Preoperative BCVA was better than 20/40 in 377 eyes (61.9\%), 20/40-20/80 in 157 eyes (25.8\%), and worse than $20 / 100$ in 75 eyes (12.3\%). Postoperative BCVA was better than 20/40 in 590 eyes (96.7\%), 20/40-20/80 in 12 eyes $(2.0 \%)$, and worse than $20 / 100$ in 7 eyes $(1.1 \%)$. The low visual acuities found in the 7 patients were related to AMD, 

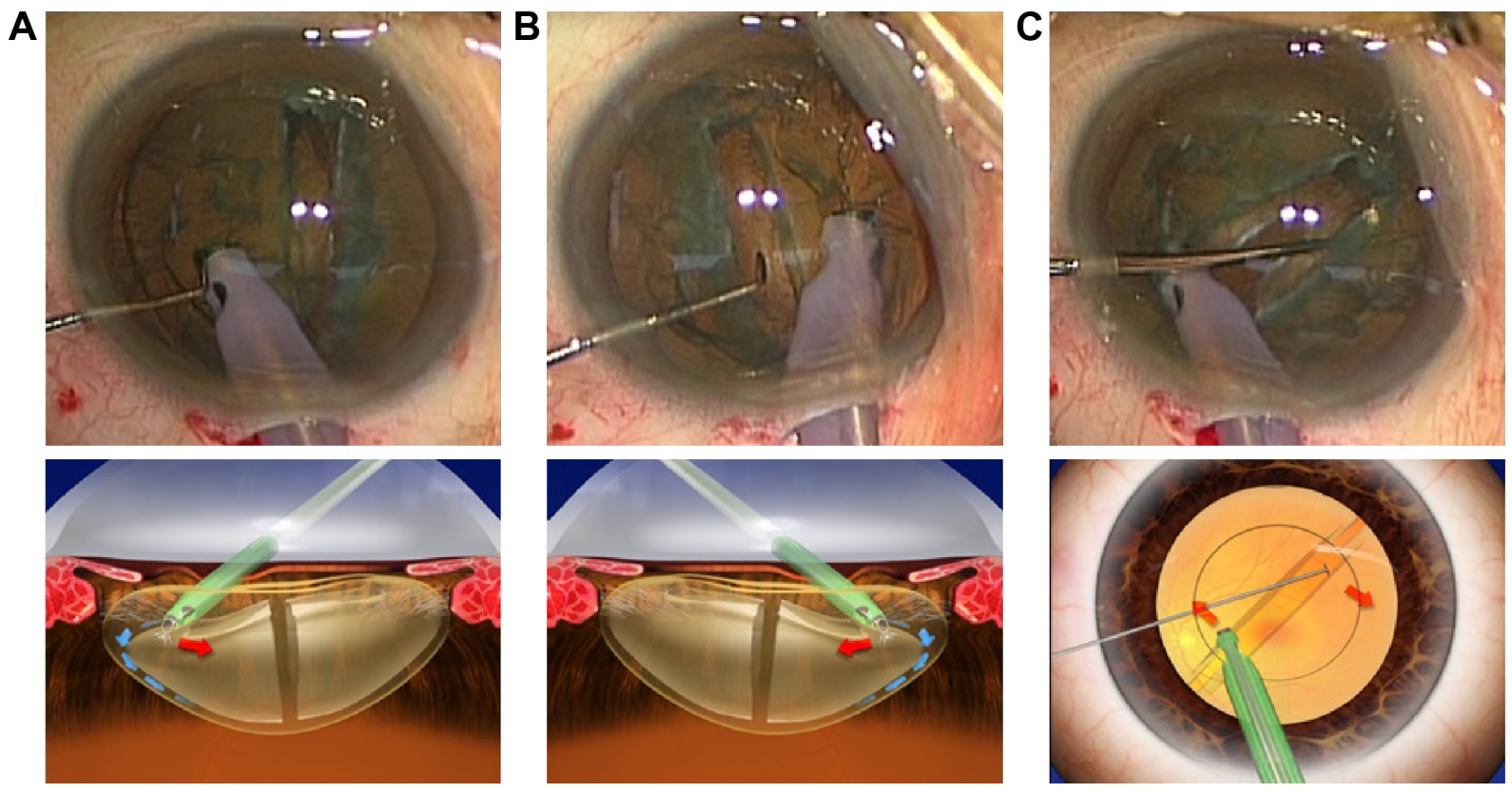

Figure 4 Anterior $\mathrm{iH}$ after posterior cleavage, if needed.

Notes: (A upper and lower) The phacoemulsification tip is placed under the left capsulorhexis edge, with anterior cortical-capsular iH then performed on the left hemi-nucleus in order to free the cortical-capsular connection. (B upper and lower) Anterior cortical-capsular iH is performed on the right hemi-nucleus, in order to free the right hemi-nucleus. (C upper and lower) Following the posterior and anterior cortical-capsular $\mathrm{iH}$, the lens is freed from the cortical-capsular connection and can be rotated circumferentially by the phacoemulsification tip for removal by a hook. The schematic illustration shows the direction of the movement of the lens segments caused by the instruments (red arrows), the vacuuming of the aqueous (white lines), and the irrigation flow (blue arrows).

Abbreviation: iH, irrigation-hydro.

diabetic retinopathy, epiretinal membrane, glaucoma, retinal vein occlusion, optic neuropathy, and amblyopia.

\section{Discussion}

The iH technique presented here is used for cortical-capsular cleavage during phacoemulsification cataract surgery. The mechanism underlying this technique involves the use of the irrigation dynamic pressure derived from the phaco tip sleeve to cleave the cortical-capsular connection. Therefore, the key element in this technique is the intentional induction of the irrigation flow from the tip sleeve within the endocapsular space; that is, elimination of the intraocular fluid from the endocapsular space. As a result, this induces an irrigation flow that compensates for the volume of the intraocular fluid that is lost during the procedure. Therefore, we intentionally removed the intraocular fluid via the phaco tip and then used it to induce the irrigation flow from the sleeve. The net result of this action is that there is a reduction in the IOP during the iH procedure. Furthermore, as the IOP does not increase above the irrigation bottle-height-dependent hydrostatic pressure, it is then possible to perform cortical-capsular hydrodissection by using the irrigation flow within the range of the expected peak IOP. In addition, we can perform the $\mathrm{iH}$ without any absorption and/or rupture of the capsule, as the nucleus stretches the capsule centrifugally and the ultrasound power is zero.

The utility of the $\mathrm{iH}$ technique was shown in 607 of 609 consecutive cases, with the procedure performed without complications in cases with pseudoexfoliation syndrome, phacodonesis, brunescent/white cataract, small pupil, shallow anterior chamber, and long or short ocular axial length, all of which are considered to be high risks in cataract surgery. ${ }^{15,16}$

Complications associated with the manual-hydro procedure are caused by stress due to high pressure within the endocapsular space, capsule-zonule complex, and posterior chamber-anterior hyaloid membrane barrier area. ${ }^{4-14}$ The $\mathrm{iH}$ technique may be able to solve these problems, as the intraocular peak pressure depends on the bottle-height hydrostatic pressure, which does not lead to the development of additional excessive pressure during the phacoemulsification surgery. Capsular block syndrome induced by the manualhydro procedure during femtosecond laser-assisted cataract surgery (FLACS) has also recently been reported to occur because of the expansion of the endocapsular volume by the laser-induced intracapsular gas and cortical changes. ${ }^{9}$ In cases with FLACS, it is important to reduce the intracapsular gas before the hydrodissection procedure in order to avoid 
high-pressure complications. Thus, the vertical groove sculpture procedure that is performed in $\mathrm{iH}$ might reduce the intracapsular gas and be safer for FLACS as compared to the manual-hydro procedure. Since phacoemulsification techniques now tend to use smaller incisions, ${ }^{17}$ high-pressure hydrodissection-related complications may occur more frequently due to this decreased incision size. Furthermore, in order to reduce these high-pressure complications, several different methods are now being used instead of the manual-hydro procedure. ${ }^{18,19}$ In addition, when using the iH technique, cataract surgery can be performed without encountering the potential high pressures that occur during the manual-hydro procedure.

\section{Conclusion}

In conclusion, the $\mathrm{iH}$ technique was developed for the purpose of separating the cortical-capsular connection in order to permit the removal of the lens. This technique uses a bottle-height-dependent limited peak pressure, which may contribute to avoidance of high-pressure complications. This technique also utilizes common phacoemulsification instruments, which should permit the straightforward adoption of the $\mathrm{iH}$ technique by experienced cataract surgeons.

\section{Acknowledgments}

We thank Toshinori Okamoto, Kunihiro Asakawa, and Akiko Kiriyama for their helpful suggestions regarding this technique.

\section{Disclosure}

The authors report no conflicts of interest in this work.

\section{References}

1. Faust KJ. Hydrodissection of soft nuclei. Am Intra-Ocular Implant Soc J. 1984;10(1):75-77.

2. Fine IH. Cortical cleaving hydrodissection. $J$ Cataract Refract Surg. 1992;18(5):508-512.
3. Varsavada AR, Singh R, Apple DJ, Trivedi RH, Pandey SK, Werner L. Effect of hydrodissection on intraoperative performance: randomized study. J Cataract Refract Surg. 2002;28(9):1623-1628.

4. Khng C, Packer M, Fine IH, Hoffman RS, Moreira FB. Intraocular pressure during phacoemulsification. J Cataract Refract Surg. 2006; 32(2):301-308.

5. Kawasaki S, Suzuki T, Yamaguchi M, et al. Disruption of the posterior chamber-anterior hyaloid membrane barrier during phacoemulsification and aspiration as revealed by contrast-enhanced magnetic resonance imaging. Arch Ophthalmol. 2009;127(4):465-470.

6. Kawasaki S, Tasaka Y, Suzuki T, et al. Influence of elevated intraocular pressure on the posterior chamber-anterior hyaloid membrane barrier during cataract operations. Arch Ophthalmol. 2011;129(6):751-757.

7. Ota I, Miyake S, Miyake K. Dislocation of the lens nucleus into the vitreous cavity after standard hydrodissection. Am J Ophthalmol. 1996;121(6):706-708.

8. Miyake K, Ota I, Ichihashi S, Miyake S, Tanaka Y, Terasaki H. New classification of the capsular block syndrome. J Cataract Refract Surg. 1998;24(9):1230-1234.

9. Roberts TV, Sutton G, Lawless MA, Jindal-Bali S, Hodge C. Capsular block syndrome associated with femtosecond laser-assisted cataract surgery. J Cataract Refract Surg. 2011;37(11):2068-2070.

10. Hurvitz LM. Posterior capsular rupture at hydrodissection (letter). $J$ Cataract Refract Surg. 1991;17(6):866.

11. Osher RH, Yu BC, Koch DD. Posterior polar cataracts: a predisposition to intraoperative posterior capsular rupture. J Cataract Refract Surg. 1990;16(2):157-162.

12. Marques FF, Marques DMV, Osher RH, Osher JM. Fate of anterior capsule tears during cataract surgery. J Cataract Refract Surg. 2006; 32(10):1638-1642.

13. Mackool RJ, Sirota M. Infusion misdirection syndrome. J Cataract Refract Surg. 1993;19(6):819.

14. Gryzmowski A, Prasad S. Acute aqueous misdirection syndrome: pathophysiology and management. J Cataract Refract Surg. 2014; 40(12):2167.

15. Gupta A, Singh J, Dhillon B. Cataract classification system for risk stratification in surgery. J Cataract Refract Surg. 2011;37(7):1363-1364.

16. Butler TK. Risk stratification and assessment in cataract surgery. $J$ Cataract Refract Surg. 2012;38(1):184.

17. Tsuneoka H, Shiba T, Takahashi Y. Ultrasonic phacoemulsification using a $1.4 \mathrm{~mm}$ incision: clinical results. $J$ Cataract Refract Surg. 2002;28(1):81-86.

18. Masuda Y, Tsuneoka H. Hydrodissection-free phacoemulsification surgery: mechanical cortical cleaving dissection. $J$ Cataract Refract Surg. 2014;40(8):1327-1331.

19. Soda M, Yaguchi S. Phacoemulsification without hydrodissection: semi-crater and split technique. J Cataract Refract Surg. 2015; 41(6):1132-1136
Clinical Ophthalmology

\section{Publish your work in this journal}

Clinical Ophthalmology is an international, peer-reviewed journal covering all subspecialties within ophthalmology. Key topics include: Optometry; Visual science; Pharmacology and drug therapy in eye diseases; Basic Sciences; Primary and Secondary eye care; Patient Safety and Quality of Care Improvements. This journal is indexed on Submit your manuscript here: http://www.dovepress.com/clinical-ophthalmology-journal

\section{Dovepress}

PubMed Central and CAS, and is the official journal of The Society of Clinical Ophthalmology (SCO). The manuscript management system is completely online and includes a very quick and fair peer-review system, which is all easy to use. Visit http://www.dovepress.com/ testimonials.php to read real quotes from published authors. 